\title{
Clean Database
}

National Cancer Institute

\section{Source}

National Cancer Institute. Clean Database. NCI Thesaurus. Code C142419.

A database in which all errors have been detected and resolved. 